\title{
Experimentelle Untersuchungen über den Einfluss des Alkohols auf den Kohlehydratstofiwechsel.
}

\author{
II. Mitteilung. Über die schädliche Wirkung des Alkohols \\ gegen die Glukosebehandlung bei verschiedenen \\ experimentellen Leberschäden.
}

von

\section{Huzio Matunaga.}

(松 永藤 雄)

\section{(Aus der Medizinischen Klinik von Prof. T. Kurokawa der Medizinischen Fakultät der Tōhoku \\ Reichsuniversitüt, Sendai.)}

\section{Einleitung.}

Wie es in der I. Mitteilung ${ }^{1)}$ dieser Arbeit zusammenfassend erörtert wurde, fand die Alkoholbehandlung in der medizinischen Praxis umfangreiche Anwendung, bald als Nahrungsmittel in der Diätetik, bald als schmerzstillendes Mittel, bald als Desinfizienz gegen eitrige oder jauchige Prozesse. In bezug auf letzt genannte Anwendung sind früher schon verschiedene Mitteilungen erschienen.

Th urs $\mathrm{z}^{2}$ ist der erste, der intravenöse Injektion ziemlich grosser Dose Alkohols (200 ccm 33\%) gegen septische Erscheinung infolge jauchenden Carzinoms einer Patientin ohne nennenswerte Nebenwirkung ausgeführt hat. Einige Jahre später wurde diese Methode in Frankreich von Landau, Feijin und Bauer ${ }^{3)}$ zur Behandlung von Lungengangrän übernommen. Der Erfolg der Alkoholinfusion nach der Thursz schen Methode wurde von Marino $0^{4)}$, Brüll ${ }^{5)}$, Naga mat ${ }^{6)}$, Kin ${ }^{7)}$, Watanabe und Sano ${ }^{8)}$, Akaiwa u. Suzuki ${ }^{9)}$, Goto ${ }^{10)}$, Azuma und Matan ${ }^{11) 12)}$, 1 was a ki und $\operatorname{Sin} n \bar{o}^{13)}$ usf. durchaus bestätigt.

Wie in der I. Mitteilung gesagt, übt der Alkohol auf den Kohlehydratstoffwechsel bedeutenden Einfluss aus je nach der Stoffwechsellage des zu untersuchenden Organismus.

Auf welche Weise wirkt nun der Alkohol bei verschiedenen Leber- 
schädigungen, die bei eitrigen oder jauchenden Prozessen mehr oder minder bestehen mögen? Das ist nächstliegende Frage. Einerseits ist es schon bekannt, dass die Glukosetherapie bei Leberschäden die wirksamste ist, wie es schon seit $20 \mathrm{Jahren}$ von verschiedenen Autoren unseres Institutes veröffentlicht wurde. Um zu untersuchen, ob der Alkohol mit Kombination von Glukose bei experimentell hergestellter Leberschädigung ohne Beeinträchtigung des Kohlehydratstoffwechsels durchzuführen ist oder nicht, habe ich diese Untersuchung vorgenommen.

\section{Versuchsmethodik.}

Die Versuchsanordnung in vorliegender Untersuchung gestaltete sich genau so wie bei der I. Mitteilung dieser Arbeit.

Als Lebergift zog ich 3 Substanzen heran: d.h. Typhusvakzin als Endotoxin, Diphtherietoxin als Exotoxin und Chloroform als chemisches Gift. Über die Einzelheiten der Leberschädigung bespreche ich weiter in den betreffenden Kapiteln.

\section{Versuchsergebnisse.}

(A) Versuche bei den mit Typhusvakzin vergifteten Kaninchen.

\section{Kontrollversuche.}

Als Typhusvakzin wählte ich "Typhoid vaccin", welches aus dem Institut für Infektionskrankheiten in der Reichsuniversität zu Tōkyō hergestellt wurde. $0,5 \mathrm{bzw} .1,0 \mathrm{ccm}$ der Vakzinlösung pro $\mathrm{kg}$ wurden den Kaninchen subcutan injiziert, die 56 Stunden lang unter vollkommener Speiseenthaltung blieben und dann mit Glukose bzw. GlukoseAlkohol wie üblich behandelt wurden. 4 Stunden nach der letzten Behandlung, d.h. 60 Stunden später vom Zeitpunkte der Vakzininjektion, wurden die Tiere getötet, um den Gehalt der Leber an Glykogen zu analysieren.

Die Kontrolltiere, welche ohne Vakzininjektion 56 Stunden lang nur unter Nahrungsentzug gesetzt und dann mit Glukose allein behandelt wurden, enthielten 2,950\% Leberglykogen (Tabelle 1). Durch Alkoholzugabe von $1 \mathrm{ccm}$ pro $\mathrm{kg}$ bei solchen Kaninchen fand man den Glykogengehalt in dem sehr vermindertem Masse von 1,278\% und durch $3 \mathrm{ccm}$ nur 0,869\% (Tabelle 2 und 3 ).

Es ist also leicht erklärlich, dass der Alkohol bei Kaninchen, die über 3 Tage ohne Nahrung waren und dann mit Glukose gemästet wurden, auf Glykogendepots der Leber ziemlich stark beeinträchtigend 
einwirkt, was schon bei den Lezithin- und Thyroxinkaninchen nach der ersten Mitteilung dieser Arbeit der Fall war.

\section{Tabelle 1.}

Glykogengehalt der Leber der 56 Stunden lang gehungerten Kaninchen. 4 Stunden nach $5 \mathrm{~g}$ Glukose pro $\mathrm{kg}$.

\begin{tabular}{|c|c|c|c|c|c|c|}
\hline \multirow{2}{*}{$\begin{array}{c}\text { Versuchs- } \\
\text { tier- } \\
\text { Nr. }\end{array}$} & \multicolumn{2}{|c|}{ Körpergewicht (g) } & \multirow{2}{*}{$\begin{array}{c}\text { Körper- } \\
\text { gewichts- } \\
\text { abnahme } \\
(\%)\end{array}$} & \multicolumn{3}{|c|}{ Leber } \\
\hline & $\begin{array}{c}\begin{array}{c}\text { vor Speise- } \\
\text { verbot }\end{array} \\
\end{array}$ & $\begin{array}{c}\text { vor } \\
\text { Glukose }\end{array}$ & & $\begin{array}{l}\text { Gewicht } \\
\text { (g) }\end{array}$ & $\mid \begin{array}{c}\text { pro kg K. G. } \\
(\mathrm{g})\end{array}$ & $\begin{array}{c}\text { Glykogen } \\
(\%)\end{array}$ \\
\hline $\begin{array}{l}1 \\
2 \\
3 \\
\end{array}$ & $\begin{array}{l}1730 \\
1640 \\
2090\end{array}$ & $\begin{array}{l}1530 \\
1500 \\
1880 \\
\end{array}$ & $\begin{array}{r}11,6 \\
8,5 \\
10,0 \\
\end{array}$ & $\begin{array}{l}44 \\
38 \\
62 \\
\end{array}$ & $\begin{array}{l}28,8 \\
25,3 \\
33,0 \\
\end{array}$ & $\begin{array}{l}2,950 \\
2,511 \\
3,390\end{array}$ \\
\hline \multicolumn{3}{|c|}{ Durchschnitt } & 10,0 & & 29,0 & 2,950 \\
\hline
\end{tabular}

Tabelle 2.

Derselbe Versuch. 4 Stunden nach $5 g$ Glukose und $1 \mathrm{ccm}$ Alkohol pro $\mathrm{kg}$.

\begin{tabular}{|c|c|c|c|c|c|c|}
\hline \multirow{2}{*}{$\begin{array}{c}\text { Versuchs- } \\
\text { tier- } \\
\text { Nr. }\end{array}$} & \multicolumn{2}{|c|}{ Körpergewicht (g) } & \multirow{2}{*}{$\begin{array}{l}\text { Körper- } \\
\text { gewichts- } \\
\text { abnahme } \\
(\%)\end{array}$} & \multicolumn{3}{|c|}{ Leber } \\
\hline & $\begin{array}{c}\text { vor Speise- } \\
\text { verbot }\end{array}$ & $\begin{array}{c}\text { vor } \\
\text { Glukose- } \\
\text { Alkohol }\end{array}$ & & $\begin{array}{c}\text { Gewicht } \\
\text { (g) }\end{array}$ & $\underset{(\mathrm{g})}{\operatorname{pro} \mathrm{kg} \text { K. G. }}$ & $\begin{array}{c}\text { Glykogen } \\
(\%)\end{array}$ \\
\hline $\begin{array}{l}4 \\
5 \\
6\end{array}$ & $\begin{array}{l}1580 \\
1980 \\
1510\end{array}$ & $\begin{array}{l}1370 \\
1780 \\
1370\end{array}$ & $\begin{array}{r}13,3 \\
10,1 \\
9,3\end{array}$ & $\begin{array}{l}32 \\
56 \\
48\end{array}$ & $\begin{array}{l}23,4 \\
31,4 \\
35,0\end{array}$ & $\begin{array}{l}0,909 \\
1,570 \\
1,355\end{array}$ \\
\hline \multicolumn{3}{|c|}{ Durchschnitt } & 10,9 & & 29,9 & 1,278 \\
\hline
\end{tabular}

Tabelle 3.

Derselbe Versuch. 4 Stunden nach $5 \mathrm{~g}$ Glukose und $3 \mathrm{ccm}$ Alkohol pro kg.

\begin{tabular}{|c|c|c|c|c|c|c|}
\hline \multirow[b]{2}{*}{$\begin{array}{c}\text { Versuehs- } \\
\text { tier- } \\
\text { Nr. }\end{array}$} & \multicolumn{2}{|c|}{ Körpergewicht(g) } & \multirow{2}{*}{$\begin{array}{c}\text { Körper- } \\
\text { gewichts- } \\
\text { abnahme } \\
(\%)\end{array}$} & \multicolumn{3}{|c|}{ Leber } \\
\hline & $\begin{array}{c}\text { vor Speise- } \\
\text { verbot }\end{array}$ & $\begin{array}{c}\text { vor } \\
\text { Glakose- } \\
\text { Alkohol }\end{array}$ & & $\begin{array}{c}\text { Gewicht } \\
(\mathrm{g})\end{array}$ & pro $\underset{(g)}{\text { kg } \mathbf{K} . \mathbf{G} .}$ & $\underset{(\%)}{\text { Glykogen }}$ \\
\hline $\begin{array}{l}7 \\
8 \\
9\end{array}$ & $\begin{array}{l}1890 \\
1630 \\
1970\end{array}$ & $\begin{array}{l}1610 \\
1380 \\
1750\end{array}$ & $\begin{array}{l}14,8 \\
15,3 \\
11,2\end{array}$ & $\begin{array}{l}54 \\
43 \\
56\end{array}$ & $\begin{array}{l}33,5 \\
31,2 \\
32,0\end{array}$ & $\begin{array}{l}0,750 \\
0,836 \\
1,020\end{array}$ \\
\hline \multicolumn{3}{|c|}{ Durchschnitt } & 13,8 & & 32,2 & 0,869 \\
\hline
\end{tabular}

2. Versuche mit $0,5 \mathrm{~cm}$ Vakzin pro $\mathrm{kg}$.

Unter dieser Dose des Vakzins betrug das Leberglykogen 4 Stunden nach Glukosedarreichung 2, 177\% (Tabelle 4), das sich, was ganz natürlich scheint, um $0,773 \%$ niedriger als bei Kontrolltieren ohne Vakzin stellte. Eine solche Differenz ist sicher auf geschädigte Leberfunktion durch Toxininjektion zurückzuführen.

Unter Zusatz von $1 \mathrm{ccm}$ Alkohol pro $\mathrm{kg}$ bei den Vakzinglukose- 
Tabelle 4.

Glykogengehalt der Leber der mit 0,5 ecm Typhusvakzin pro $\mathrm{kg}$ vergifteten Tiere 4 Stunden nach $5 \mathrm{~g}$ Glukose pro $\mathrm{kg}$.

\begin{tabular}{|c|c|c|c|c|c|c|}
\hline \multirow{2}{*}{$\begin{array}{c}\text { Versuchs- } \\
\text { tier- } \\
\text { Nr. }\end{array}$} & \multicolumn{2}{|c|}{ Körpergewicht $(g)$} & \multirow{2}{*}{$\begin{array}{l}\text { Körper- } \\
\text { gewichts- } \\
\text { abnahme } \\
(\%)\end{array}$} & \multicolumn{3}{|c|}{ Leber } \\
\hline & $\begin{array}{l}\text { vor Toxin- } \\
\text { injektion }\end{array}$ & $\begin{array}{c}\text { vor } \\
\text { Glukose }\end{array}$ & & $\begin{array}{c}\text { Gewicht } \\
(g)\end{array}$ & $\begin{array}{c}\text { pro } \mathbf{k g ~ K} \text {. G. } \\
(\mathrm{g})\end{array}$ & $\begin{array}{c}\text { Glykogen } \\
(\%)\end{array}$ \\
\hline 10 & 1610 & 1400 & 13,0 & 32 & 22,8 & 1,965 \\
\hline 11 & 1570 & 1420 & 9,6 & 33 & 23,2 & 2,425 \\
\hline 12 & 1400 & 1210 & 13,5 & 33 & 27,2 & 2,140 \\
\hline \multicolumn{3}{|c|}{ Durchschnitt } & 12,0 & & 24,4 & 2,177 \\
\hline
\end{tabular}

kaninchen betrug das Leberglykogen nur 0,997\%. Weiter, unter 3 ccm Alkohol erzielte ich nur 0,520\%, d.i. ungefähr ein Viertel des Glykogens bei Tieren ohne Alkoholhinzufügung (Tabelle 5 und 6). Diese Verminderung des Leberglykogens beträgt, im Vergleich zu den entsprechenden Ergebnissen der Tiere ohne Vakzinbehandelung, je ungefähr $50 \%$ (vgl. Tabelle 2 und 3).

Tabelle 5 .

Derselbe Versuch. 4 Stunden nach $5 \mathrm{~g}$ Glukose und 1 ecm Alkohol pro kg.

\begin{tabular}{|c|c|c|c|c|c|c|}
\hline \multirow{2}{*}{$\begin{array}{c}\text { Versuchs- } \\
\text { tier- } \\
\text { Nr. }\end{array}$} & \multicolumn{2}{|c|}{ Körpergewicht (g) } & \multirow{2}{*}{$\begin{array}{c}\text { Körper- } \\
\text { gewichts- } \\
\text { abnahme } \\
(\%)\end{array}$} & \multicolumn{3}{|c|}{ Leber } \\
\hline & $\begin{array}{l}\text { vor Toxin- } \\
\text { injektion }\end{array}$ & $\begin{array}{c}\text { yor } \\
\text { Glukose- } \\
\text { Alkohol }\end{array}$ & & $\begin{array}{c}\text { Gewicht } \\
(\mathrm{g})\end{array}$ & $|\underset{(\mathrm{g})}{\operatorname{pro} \mathbf{k g} \mathbf{K} . \mathbf{G} .}|$ & $\begin{array}{c}\text { Glykogen } \\
(\%)\end{array}$ \\
\hline 13 & 1580 & 1280 & 19,0 & 45 & 35,2 & 0,611 \\
\hline 14 & 1480 & 1320 & 10,8 & 36 & 27,2 & 1,156 \\
\hline 15 & 1780 & 1620 & 9,0 & 42 & 25,9 & 1,225 \\
\hline \multicolumn{3}{|c|}{ Durchschnitt } & 12,9 & & 29,4 & 0,997 \\
\hline
\end{tabular}

Tabelle 6.

Derselbe Versuch. 4 Stunden nach $5 \mathrm{~g}$ Glukose und $3 \mathrm{ccm}$ Alkohol pro $\mathrm{kg}$.

\begin{tabular}{|c|c|c|c|c|c|c|}
\hline \multirow[b]{2}{*}{$\begin{array}{c}\text { Versuchs- } \\
\text { tier- } \\
\text { Nr. }\end{array}$} & \multicolumn{2}{|c|}{ Körpergewicht (g) } & \multirow{2}{*}{$\begin{array}{c}\text { Körper- } \\
\text { gewichts- } \\
\text { abnahme } \\
(\%)\end{array}$} & \multicolumn{3}{|c|}{ Leber } \\
\hline & $\begin{array}{l}\text { vor Toxin- } \\
\text { injektion }\end{array}$ & $\begin{array}{l}\text { vor } \\
\text { Glukose- } \\
\text { Alkohol }\end{array}$ & & $\begin{array}{l}\text { Gewicht } \\
\text { (g) }\end{array}$ & $\begin{array}{c}\text { pro } \underset{\text { kg K. G. G. }}{(\mathbf{g})} \\
\text {. }\end{array}$ & $\begin{array}{c}\text { Glykogen } \\
(\text { \%o) }\end{array}$ \\
\hline 16 & 1835 & 1600 & 12,8 & 54 & 33,8 & 0,820 \\
\hline 17 & 1550 & 1350 & 12,9 & 37 & 37,4 & 0,400 \\
\hline 18 & 1460 & 1350 & 7,3 & 36 & 26,6 & 0,340 \\
\hline \multicolumn{3}{|c|}{ Durehschnitt } & 11,0 & & 29,3 & $\mathbf{0 , 5 2 0}$ \\
\hline
\end{tabular}

Hieraus kann man wohl schliessen, dass diese Abnahme des Glykogengehalts auf Summation der schädlichen Wirkung des Typhusvakzins und des Alkohols auf die Fixation des Glykogens in der Leber beruht. 
3. Versuche mit 1,0cm Vakzin pro $\mathrm{kg}$.

Unter zweifach vergrösserter Vakzinmenge fand man bei Glukosekaninchen 1,018\% Leberglykogen, was einem Drittel von Tieren ohne Vakzin und der Hälfte von denen mit $0,5 \mathrm{ccm}$ Vakzin belasteten entspricht (Tabelle 7); also geht der Glykogenansatz nach Glukosedarrei. chung entsprechend der Zunahme der Vakzindose herab.

Tabelle 7.

Glykogengehalt der Leber der mit 1,0 com Typhusvakzin pro $\mathrm{kg}$ vergifteten Tiere. 4 Stunden nach $5 \mathrm{~g}$ Glukose pro $\mathrm{kg}$.

\begin{tabular}{|c|c|c|c|c|c|c|}
\hline \multirow{2}{*}{$\begin{array}{c}\text { Versuchs- } \\
\text { tier- } \\
\text { Nr. }\end{array}$} & \multicolumn{2}{|c|}{ Körpergewicht (g) } & \multirow{2}{*}{$\begin{array}{c}\text { Körper- } \\
\text { gewichts- } \\
\text { abnahme } \\
(\%)\end{array}$} & \multicolumn{3}{|c|}{ Leber } \\
\hline & $\begin{array}{l}\text { vor Toxin- } \\
\text { injektion }\end{array}$ & $\begin{array}{c}\text { vor } \\
\text { Glukose }\end{array}$ & & $\begin{array}{c}\text { Gewicht } \\
(\mathrm{g})\end{array}$ & $\begin{array}{c}\text { pro kg K. G. } \\
(\mathrm{g})\end{array}$ & $\begin{array}{c}\text { Glykogen } \\
(\%)\end{array}$ \\
\hline $\begin{array}{l}19 \\
20 \\
.21\end{array}$ & $\begin{array}{l}1960 \\
1310 \\
1540\end{array}$ & $\begin{array}{l}1710 \\
1050 \\
1220\end{array}$ & $\begin{array}{l}12,7 \\
19,8 \\
20,8\end{array}$ & $\begin{array}{l}52 \\
28 \\
28\end{array}$ & $\begin{array}{l}30,4 \\
26,6 \\
23,0\end{array}$ & $\begin{array}{l}1,300 \\
0,855 \\
0,900\end{array}$ \\
\hline \multicolumn{2}{|c|}{ Durchschnitt } & - & 17,8 & & 26,3 & 1,018 \\
\hline
\end{tabular}

Dann verfolgte ich weiter die Veränderung des Glykogenvorrats unter Hinzufügung von $1 \mathrm{ccm}$ bzw. $3 \mathrm{ccm}$ Alkohol pro $\mathrm{kg}$. Bei diesen beiden Fällen betrug die ermittelte Glykogenmenge je 0,847 bzw. 0,492\% (Tabelle 8 und 9).

\section{Tabelle 8.}

Derselbe Versuch. 4 Stunden nach $5 \mathrm{~g}$ Glukose und $1 \mathrm{ccm}$ Alkohol pro $\mathrm{kg}$.

\begin{tabular}{|c|c|c|c|c|c|c|}
\hline \multirow[b]{2}{*}{$\begin{array}{c}\text { Versuchs- } \\
\text { tier- } \\
\mathrm{Nr} .\end{array}$} & \multicolumn{2}{|c|}{ Körpergewicht(g) } & \multirow{2}{*}{$\begin{array}{c}\text { Körper- } \\
\text { gewichts- } \\
\text { abnahme } \\
(\%)\end{array}$} & \multicolumn{3}{|c|}{ Leber } \\
\hline & $\begin{array}{l}\text { vor Toxin- } \\
\text { injektion }\end{array}$ & $\begin{array}{c}\text { vor } \\
\text { Glukose- } \\
\text { Alkohol }\end{array}$ & & $\begin{array}{c}\text { Gewicht } \\
\text { (g) }\end{array}$ & $\underset{(\mathbf{g})}{\text { pro } \mathbf{k g} \text { K. G. }}$ & $\begin{array}{l}\text { Glykogen } \\
(\%)\end{array}$ \\
\hline $\begin{array}{l}22 \\
23 \\
24\end{array}$ & $\begin{array}{l}1640 \\
1400 \\
1640\end{array}$ & $\begin{array}{l}1400 \\
1230 \\
1380\end{array}$ & $\begin{array}{l}14,6 \\
12,1 \\
15,8\end{array}$ & $\begin{array}{l}45 \\
40 \\
35\end{array}$ & $\begin{array}{l}32,2 \\
32,5 \\
25,4\end{array}$ & $\begin{array}{l}0,860 \\
0,820 \\
0,860\end{array}$ \\
\hline \multicolumn{3}{|c|}{ Durchsehnitt } & 14,2 & & 30,0 & 0,847 \\
\hline
\end{tabular}

Tabelle 9.

Derselbe Versuch. 4 Stunden nach $5 \mathrm{~g}$ Glukose und 3 ccm Alkohol pro kg.

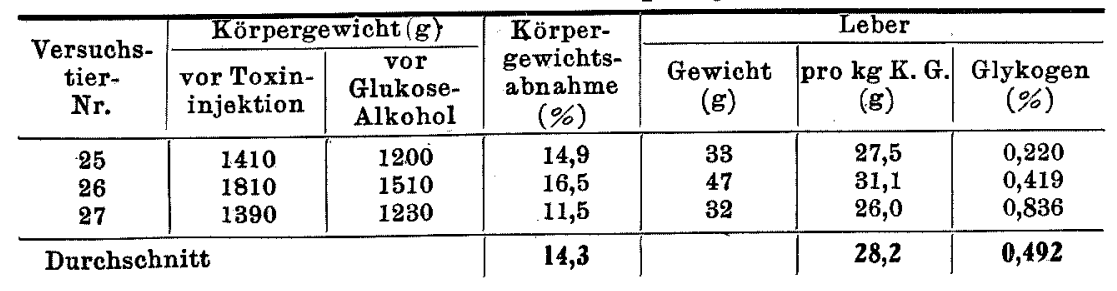


Daraus kann man schliessen, dass das Leberglykogen, welches von zugeführter Glukose hergestellt ist, unter Hinzufügung von Alkohol vermindert gefunden wird, und zwar je mehr der Alkohol zugesetzt wird, desto weniger wird das Glykogen aus Glukose neugebildet.

\section{(B) Versuche bei den mit Diphtherietoxin vergifteten Kaninchen.}

Das Diphtherietoxin, welches zum Versuch als Exotoxin herangezogen wurde, wurde im Institut für Infektionskrankheiten der Reichsuniversität zu Tōkyō so hergestellt, dass die M.L.D. dieses Toxins $1 / 1000 \mathrm{~cm}$ war. Unter Verdünnung von 1:500 mit physiologischer Kochsalzlösung gebrauchte ich $0,3 \mathrm{ccm}$ bzw. $0,7 \mathrm{ccm}$ dieses Toxins pro $\mathrm{kg}$ bei den normal gefütterten Kaninchen intravenös.

56 Stunden nach Injektion erhielten die Tiere die Probemahlzeit von Glukose bzw. Glukose-Alkohollösung wie üblich und wurden 4 Stunden später getötet, um den Glykogengehalt der Leber zu ermitteln.

\section{Versuche mit $0,3 \mathrm{ccm}$ verdünntem Toxin pro $\mathrm{kg}$.}

Bei Tieren, welche mit dieser kleineren Dose von verdünntem Diphtherietoxin vergiftet wurden, konnte ich 1,298\% vom Leberglykogen nach Darreichung von $5 \mathrm{~g}$ Glukose finden (Tabelle 10). Bei Zusatz von $1 \mathrm{bzw}$. $3 \mathrm{ccm}$ Alkohol pro kg zu Glukose betrug Leberglykogen je nach der Alkoholdose 0,923 bzw. 0,819\% (Tabelle 11 und 12).

Vergleicht man die Daten der Toxinkaninchen mit denen der Typhusvakzintiere, so scheint mir die Glukoseassimilation bei den ersteren ziemlich schlechter als bei den letzteren zu sein, während die Wirkung des Alkohols auf den Glykogengehalt der Leber bei jenen weniger beeinträchtigend als bei diesen ist.

Tabelle 10.

Glykogengehalt der Leber der mit 0,3 ccm pro $\mathrm{kg}$ von 500 fach verdünntem Diphtherietoxin vergifteten Tiere. 4 Stunden nach $5 \mathrm{~g}$ Glukose pro lkg.

\begin{tabular}{|c|c|c|c|c|c|c|}
\hline \multirow{2}{*}{$\begin{array}{c}\text { Versuchs- } \\
\text { tier- } \\
\text { Nr. }\end{array}$} & \multicolumn{2}{|c|}{ Körpergewicht (g) } & \multirow{2}{*}{$\begin{array}{l}\text { Körper- } \\
\text { gewichts- } \\
\text { abnahme } \\
(\%)\end{array}$} & \multicolumn{3}{|c|}{ Leber } \\
\hline & $\begin{array}{l}\text { vor Toxin- } \\
\text { injektion }\end{array}$ & $\begin{array}{c}\text { vor } \\
\text { Glukose }\end{array}$ & & $\begin{array}{c}\text { Gowioht } \\
\text { (g) }\end{array}$ & \begin{tabular}{|c} 
pro $\mathbf{k g ~ K . ~ G . ~}$ \\
$(\mathrm{g})$
\end{tabular} & $\begin{array}{c}\text { Glykogen } \\
(\%)\end{array}$ \\
\hline $\begin{array}{l}28 \\
29 \\
30\end{array}$ & $\begin{array}{l}1750 \\
2440 \\
2020\end{array}$ & $\begin{array}{l}1540 \\
2090 \\
1760\end{array}$ & $\begin{array}{l}12,0 \\
14,3 \\
12,9\end{array}$ & $\begin{array}{l}49 \\
62 \\
61\end{array}$ & $\begin{array}{l}31,8 \\
29,7 \\
34,7\end{array}$ & $\begin{array}{l}1,789 \\
1,006 \\
1,100\end{array}$ \\
\hline \multicolumn{3}{|c|}{ Durchschnitt } & 13,1 & & 32,1 & 1,298 \\
\hline
\end{tabular}


Tabelle 11.

Derselbe Versuch. 4 Stunden nach $5 \mathrm{~g}$ Glukose und $1 \mathrm{ccm}$ Alkohol pro $\mathrm{kg}$.

\begin{tabular}{|c|c|c|c|c|c|c|}
\hline \multirow{2}{*}{$\begin{array}{l}\text { Versuchs- } \\
\text { tier- } \\
\text { Nr. }\end{array}$} & \multicolumn{2}{|c|}{ Körpergewicht (g) } & \multirow{2}{*}{$\begin{array}{c}\text { Körper- } \\
\text { gewichts- } \\
\text { abnahme } \\
(\%) .\end{array}$} & \multicolumn{3}{|c|}{ Leber } \\
\hline & $\begin{array}{l}\text { vor Toxin- } \\
\text { injektion }\end{array}$ & $\begin{array}{c}\text { vor } \\
\text { Glukose- } \\
\text { Alkohol }\end{array}$ & & $\begin{array}{c}\text { Gewicht } \\
\text { (g) }\end{array}$ & $\begin{array}{c}\text { pro kg K. G. } \\
(\mathbf{g})\end{array}$ & $\begin{array}{c}\text { Glykogen } \\
(\%)\end{array}$ \\
\hline $\begin{array}{l}31 \\
32 \\
33\end{array}$ & $\begin{array}{l}1730 \\
1860 \\
2440 \\
\end{array}$ & $\begin{array}{l}1490 \\
1570 \\
2170 \\
\end{array}$ & $\begin{array}{l}13,9 \\
15,6 \\
11,1 \\
\end{array}$ & $\begin{array}{l}44 \\
64 \\
69 \\
\end{array}$ & $\begin{array}{l}29,5 \\
40,7 \\
31,8 \\
\end{array}$ & $\begin{array}{l}0,995 \\
0,733 \\
1,041\end{array}$ \\
\hline \multicolumn{3}{|c|}{ Durchschnitt } & 13,5 & & 34,0 & 0,923 \\
\hline
\end{tabular}

Tabelle 12.

Derselbe Versuch. I Stunden nach 5 g Glukase und $3 \mathrm{ccm}$ Alkohol pro $\mathrm{kg}$.

\begin{tabular}{|c|c|c|c|c|c|c|}
\hline \multirow[b]{2}{*}{$\begin{array}{c}\text { Versuchs- } \\
\text { tier- } \\
\text { Nr. }\end{array}$} & \multicolumn{2}{|c|}{ Körpergewicht(g) } & \multirow{2}{*}{$\begin{array}{l}\text { Körper- } \\
\text { gewichts- } \\
\text { abnahme } \\
(\%)\end{array}$} & \multicolumn{3}{|c|}{ Lueber } \\
\hline & $\begin{array}{l}\text { vor Toxin- } \\
\text { injektion }\end{array}$ & $\begin{array}{l}\text { vor } \\
\text { Glukose- } \\
\text { Alkohol }\end{array}$ & & $\begin{array}{c}\text { Gewicht } \\
(\mathrm{g})\end{array}$ & $\begin{array}{c}\text { pro kg K. G. } \\
(\mathrm{g})\end{array}$ & $\begin{array}{c}\text { Glykogen } \\
(\%)\end{array}$ \\
\hline $\begin{array}{l}34 \\
35 \\
36\end{array}$ & $\begin{array}{l}1740 \\
2210 \\
2130\end{array}$ & $\begin{array}{l}1460 \\
2010 \\
1900\end{array}$ & $\begin{array}{r}16,1 \\
9,1 \\
10,8\end{array}$ & $\begin{array}{l}52 \\
58 \\
63\end{array}$ & $\begin{array}{l}35,6 \\
28,8 \\
33,1\end{array}$ & $\begin{array}{l}0,850 \\
0,611 \\
0,995\end{array}$ \\
\hline \multicolumn{3}{|c|}{ Durchschnitt } & 12,0 & & 32,5 & 0,819 \\
\hline
\end{tabular}

2. Versuche mit $0,7 \mathrm{ccm}$ verdünntem Toxin pro $\mathrm{kg}$.

Dabei wird die Fähigkeit der Leber, Glukose zu assimilieren, im grossen und ganzen etwas mehr angegriffen als bei $0,3 \mathrm{ccm}$ Diphtherietoxin.

Nach einfacher Darreichung von $5 \mathrm{~g}$ Glukose betrug das Glykogen der Leber 1,208\%, d.i. 0,09\% weniger als bei einer kleineren Menge Toxin. Nach Hinzufügung von Alkohol vermindertesich das Glykogen noch stärker. Bei $1 \mathrm{ccm}$ Alkohol war der Glykogenwert 0,844\%, und bei $3 \mathrm{ccm}$ 0,730\% (Tabelle 14 und 15).

Tabelle 13.

Glykogengehalt der Leber der mit 0,7 ccm pro kg desjenigen Diphtherietoxins vergifteten Tiere. 4 Stunden nach $5 \mathrm{~g}$ Glukose pro $\mathrm{kg}$.

\begin{tabular}{|c|c|c|c|c|c|c|}
\hline \multirow{2}{*}{$\begin{array}{c}\text { Versuchs- } \\
\text { tier- } \\
\text { Nr. }\end{array}$} & \multicolumn{2}{|c|}{ Körpergewicht (g) } & \multirow{2}{*}{$\begin{array}{l}\text { Körper- } \\
\text { gewichts- } \\
\text { abnahme } \\
(\%)\end{array}$} & \multicolumn{3}{|c|}{ Leber } \\
\hline & $\begin{array}{l}\text { vor Toxin- } \\
\text { injektion }\end{array}$ & $\begin{array}{c}\text { vor } \\
\text { Glukose } \\
\end{array}$ & & $\begin{array}{l}\text { Gewicht } \\
\text { (g) }\end{array}$ & $\mid \begin{array}{c}\text { pro kg K. G. } \\
(\mathrm{g})\end{array}$ & $\begin{array}{c}\text { Glykogen } \\
(\%)\end{array}$ \\
\hline $\begin{array}{l}37 \\
38 \\
39\end{array}$ & $\begin{array}{l}1960 \\
1750 \\
2100\end{array}$ & $\begin{array}{l}1700 \\
1510 \\
1800\end{array}$ & $\begin{array}{l}13,3 \\
13,7 \\
14,3\end{array}$ & $\begin{array}{l}53 \\
50 \\
67\end{array}$ & $\begin{array}{l}31,1 \\
33,0 \\
37,1\end{array}$ & $\begin{array}{l}1,364 \\
1,211 \\
1,050\end{array}$ \\
\hline \multicolumn{3}{|c|}{ Durchschnitt } & 13,8 & & 33,7 & 1,208 \\
\hline
\end{tabular}


Tabelle 14:

Derselbe Versuch. 4 Stunden nach $5 \mathrm{~g}$ Glukose

und $1 \mathrm{ecm}$ Alkohol pro $\mathrm{kg}$.

\begin{tabular}{|c|c|c|c|c|c|c|}
\hline \multirow[b]{2}{*}{$\begin{array}{c}\text { Versuchs- } \\
\text { tier- } \\
\text { Nr. }\end{array}$} & \multicolumn{2}{|c|}{ Körpergewicht(g) } & \multirow{2}{*}{$\begin{array}{c}\text { Körper- } \\
\text { gewichts- } \\
\text { abnahme } \\
(\%)\end{array}$} & \multicolumn{3}{|c|}{ Leber } \\
\hline & $\begin{array}{l}\text { vor Toxin- } \\
\text { injektion }\end{array}$ & $\begin{array}{c}\text { vor } \\
\text { Glukose- } \\
\text { Alkohol }\end{array}$ & & $\begin{array}{c}\text { Gewicht } \\
\text { (g) }\end{array}$ & $\begin{array}{c}\text { pro } \mathrm{kg}, \mathrm{K}, \mathrm{G} . \\
(\mathrm{g})\end{array}$ & $\begin{array}{c}\text { Glykogen } \\
(\%)\end{array}$ \\
\hline $\begin{array}{l}40 \\
41 \\
42\end{array}$ & $\begin{array}{l}1870 \\
2110 \\
1960\end{array}$ & $\begin{array}{l}1680 \\
1770 \\
1600\end{array}$ & $\begin{array}{l}10,2 \\
16,1 \\
18,4\end{array}$ & $\begin{array}{l}55 \\
60 \\
53\end{array}$ & $\begin{array}{l}32,7 \\
33,9 \\
33,1\end{array}$ & $\begin{array}{l}1,053 \\
0,500 \\
0,980\end{array}$ \\
\hline \multicolumn{3}{|c|}{ Durehschnitt } & 14,9 & & 33,2 & 0,844 \\
\hline
\end{tabular}

Tabelle 15.

Derselbe Versuch. 4 Stunden nach $5 \mathrm{~g}$ Glukose und $3 \mathrm{ccm}$ Alkohol pro $\mathrm{kg}$.

\begin{tabular}{|c|c|c|c|c|c|c|}
\hline \multirow[b]{2}{*}{$\begin{array}{c}\text { Versuchs- } \\
\text { tier- } \\
\text { Nr. }\end{array}$} & \multicolumn{2}{|c|}{ Körpergewicht(g) } & \multirow{2}{*}{$\begin{array}{c}\text { Körper- } \\
\text { gewichts- } \\
\text { abnahme } \\
(\% 0)\end{array}$} & \multicolumn{3}{|c|}{ Leber } \\
\hline & $\begin{array}{l}\text { vor Toxin- } \\
\text { injektion }\end{array}$ & $\begin{array}{c}\text { vor } \\
\text { Glukose- } \\
\text { Alkohol }\end{array}$ & & $\begin{array}{c}\text { Gewicht } \\
\text { (g) }\end{array}$ & $\mid \begin{array}{c}\text { pro } \mathrm{kg} \mathbf{K} . \mathbf{G} . \\
(\mathrm{g})\end{array}$ & $\begin{array}{c}\text { Glykogen } \\
(\% 0)\end{array}$ \\
\hline $\begin{array}{l}43 \\
44 \\
45\end{array}$ & $\begin{array}{l}2110 \\
1930 \\
2140\end{array}$ & $\begin{array}{l}1840 \\
1620 \\
1900\end{array}$ & $\begin{array}{l}12,8 \\
16,1 \\
11,2\end{array}$ & $\begin{array}{l}60 \\
51 \\
67\end{array}$ & $\begin{array}{l}32,6 \\
31,4 \\
35,2\end{array}$ & $\begin{array}{r}0,771 \\
0,767 \\
0,653\end{array}$ \\
\hline \multicolumn{3}{|c|}{ Durchschnitt } & 13,4 & & 33,6 & $\mathbf{0 , 7 3 0}$ \\
\hline
\end{tabular}

So wirkt also der Alkohol, sei es 1 oder $3 \mathrm{ccm}$ pro $\mathrm{kg}$, bei Vergiftung mit $0,7 \mathrm{ccm}$ pro kg Toxin auf das Leberglykogen gar nicht günstig.

(C) Versuche bei den mit Chloroformvergifteten Kaninchen.

\section{Kontrollversuche.}

Chloroform, welches als eines der chemischen Gifte gegen die Leber ausgewählt wurde, ruft bekanntlich eine beträchtliche Vergiftung der Leber und demzufolge einen Glykogenschwund aus dem Organ hervor. Hierüber haben schon $\mathrm{Hotta}^{15)}$, $\mathrm{Nagao}^{16)}$, Tsuda ${ }^{17)}$, Si mi$\mathrm{z} \mathrm{u}^{18)}$ u. a., in hiesiger Klinik, eine Reihe von ausführlichen Untersuchungen angestellt.

Zur Kontrolle der Chloroformtiere habe ich die entsprechenden 3 verschiedenen Versuche vorausgeschickt. Erstens wurde das Leberglykogen der Kaninchen ermittelt, die 44 Stunden lang nur gehungert, dann $5 \mathrm{~g}$ Glukose pro $\mathrm{kg}$ durch den Schlauch in den Magen erhalten hatten und 4 Stunden danach getötet wurden. Zweitens genau dieselbe Versuchsanordnung mit gleichzeitiger Zulage von 1 bzw. 3 ccm Alkohol pro kg. Die erhobene Glykogenmenge war je 2,600, 2,106 und 0,935\% (Tabelle 16, 17 und 18). 
Wie aus diesen Tabellen leicht ersichtlich, wirkt der Alkohol auch dabei deutlich schädigend auf Glukoseassimilation.

Tabelle 16.

Glykogengehalt der Leber der vor der Probemahlzeit 44 Stunden lang gehungerten Kaninchen. 4 Stunden nach $5 \mathrm{~g}$ Glukose pro $\mathrm{kg}$.

\begin{tabular}{|c|c|c|c|c|c|c|}
\hline \multirow{2}{*}{$\begin{array}{c}\text { Versuchs- } \\
\text { tier- } \\
\text { Nr. }\end{array}$} & \multicolumn{2}{|c|}{ Körpergewicht(g) } & \multirow{2}{*}{$\begin{array}{l}\text { Körper- } \\
\text { gewichts- } \\
\text { abnahme } \\
(\%)^{\circ}\end{array}$} & \multicolumn{3}{|c|}{ Leber } \\
\hline & $\begin{array}{c}\text { vor Speise- } \\
\text { verbot }\end{array}$ & $\begin{array}{c}\text { vor } \\
\text { Glukose }\end{array}$ & & $\begin{array}{c}\text { Gewicht } \\
(\mathrm{g})\end{array}$ & $\begin{array}{c}\text { pro kg } \mathrm{K} . \mathrm{G} . \\
(\mathrm{g})\end{array}$ & $\begin{array}{c}\text { Glykogen } \\
(\%)\end{array}$ \\
\hline $\begin{array}{l}46 \\
47 \\
48\end{array}$ & $\begin{array}{l}2350 \\
1980 \\
1600\end{array}$ & $\begin{array}{l}2230 \\
1820 \\
1480\end{array}$ & $\begin{array}{l}5,1 \\
8,1 \\
7,5\end{array}$ & $\begin{array}{l}69 \\
58 \\
54\end{array}$ & $\begin{array}{l}31,0 \\
31,8 \\
36,5\end{array}$ & $\begin{array}{l}2,860 \\
2,620 \\
2,320\end{array}$ \\
\hline \multicolumn{3}{|c|}{ Durchschnitt } & 6,9 & & 33,1 & 2,600 \\
\hline
\end{tabular}

Tabelle 17.

Derselbe Versuch. \& Stunden nach $5 \mathrm{~g}$ Glukose und $1 \mathrm{cem}$ Alkohol pro $\mathrm{kg}$.

\begin{tabular}{|c|c|c|c|c|c|c|}
\hline \multirow[b]{2}{*}{$\begin{array}{c}\text { Versuchs- } \\
\text { tier- } \\
\text { Nr. }\end{array}$} & \multicolumn{2}{|c|}{ Körpergewicht (g) } & \multirow{2}{*}{$\begin{array}{l}\text { Körper- } \\
\text { gewichts- } \\
\text { abnahme } \\
(\%)\end{array}$} & \multicolumn{3}{|c|}{ Leber } \\
\hline & $\begin{array}{l}\text { vor Speise- } \\
\text { verbot }\end{array}$ & $\begin{array}{c}\text { vor } \\
\text { Glukose- } \\
\text { Alkohol } \\
\end{array}$ & & $\begin{array}{c}\text { Gewicht } \\
\text { (g) }\end{array}$ & $\begin{array}{c}\text { pro kg K. G. } \\
(\mathbf{g})\end{array}$ & $\begin{array}{c}\text { Glykogen } \\
(\%)\end{array}$ \\
\hline $\begin{array}{l}49 \\
50 \\
51\end{array}$ & $\begin{array}{l}2300 \\
2010 \\
1650\end{array}$ & $\begin{array}{l}2190 \\
1870 \\
1500\end{array}$ & $\begin{array}{l}4,8 \\
7,0 \\
9,1\end{array}$ & $\begin{array}{l}52 \\
50 \\
44\end{array}$ & $\begin{array}{l}23,8 \\
26,8 \\
29,3\end{array}$ & $\begin{array}{l}2,160 \\
1,778 \\
2,380\end{array}$ \\
\hline \multicolumn{3}{|c|}{ Durchschnitt } & $\mathbf{7 , 0}$ & & 26,6 & 2,106 \\
\hline
\end{tabular}

Tabelle 18.

Derselbe Versuch. 4 Stunden nach $5 \mathrm{~g}$ Glukose und $3 \mathrm{cem}$ Alkohol pro $\mathrm{kg}$.

\begin{tabular}{c|c|c|c|c|c|c}
\hline $\begin{array}{c}\text { Versuchs- } \\
\text { tier- } \\
\text { Nr. }\end{array}$ & $\begin{array}{c}\text { Körpergewicht }(\mathrm{g}) \\
\text { vor Speise- } \\
\text { verbot }\end{array}$ & $\begin{array}{c}\text { vor } \\
\text { Glukose- } \\
\text { Alkohol }\end{array}$ & $\begin{array}{c}\text { Körper- } \\
\text { gewichts- } \\
\text { abnahme } \\
(\%)\end{array}$ & $\begin{array}{c}\text { Gewicht } \\
(\mathbf{g})\end{array}$ & $\begin{array}{c}\text { pro kg K. G. } \\
(\mathbf{g})\end{array}$ & $\begin{array}{c}\text { Glykogen } \\
(\%)\end{array}$ \\
\hline $\mathbf{5 2}$ & 1880 & 1750 & 6,9 & 54 & 30,8 & 0,711 \\
$\mathbf{5 3}$ & 1510 & 1420 & 6,0 & 50 & 35,2 & 1,096 \\
$\mathbf{5 4}$ & 1710 & 1600 & 6,4 & $\mathbf{5 2}$ & $\mathbf{3 2 , 5}$ & $\mathbf{0 , 9 9 9}$ \\
\hline \multicolumn{2}{l}{ Durchschnitt } & & $\mathbf{6 , 4}$ & & $\mathbf{3 2 , 8}$ & $\mathbf{0 , 9 3 5}$
\end{tabular}

\section{Versuche mit $0,15 \mathrm{~cm}$ Chloroform pro $\mathrm{kg}$.}

24 Stunden hungernde Kaninchen erhielten $0,15 \mathrm{ccm}$ Chloroform pro $\mathrm{kg}$ subcutan, 20 Stunden danach $5 \mathrm{~g}$ Glukose pro kg per os und 4 Stunden nach der Glukosegabe wurden die Tiere getötet. Das gefundene Leberglykogen wardurchschnittlich 1,511\%, die dem Kontrollwerte ohne Giftwirkung in starkem Masse nachstehen (Tabelle 19). 


\section{Tabelle 19.}

Glykogengehalt der Leber der mit 0,15 com Chloroform pro $\mathrm{kg}$ vergifteten Tiere. 4 Stunden nach $5 \mathrm{~g}$ Glukose pro $\mathrm{kg}$.

\begin{tabular}{|c|c|c|c|c|c|c|}
\hline \multirow{2}{*}{$\begin{array}{c}\text { Versuchs- } \\
\text { tier- } \\
\text { Nr. } \\
\end{array}$} & \multicolumn{2}{|c|}{ Körpergewicht (g) } & \multirow{2}{*}{$\begin{array}{c}\text { Körper- } \\
\text { gewichts- } \\
\text { abnahme } \\
(\%) .\end{array}$} & \multicolumn{3}{|c|}{ Leber } \\
\hline & $\begin{array}{c}\begin{array}{c}\text { vor Chloro- } \\
\text { form }\end{array} \\
\end{array}$ & $\begin{array}{c}\text { vor } \\
\text { Glukose } \\
\end{array}$ & & $\begin{array}{c}\text { Gewieht } \\
\text { (g) }\end{array}$ & $\begin{array}{c}\text { pro kg K. G. } \\
(\mathrm{g})\end{array}$ & $\begin{array}{c}\text { Glykogen } \\
(\%)\end{array}$ \\
\hline $\begin{array}{l}\mathbf{5 5} \\
\mathbf{5 6} \\
\mathbf{5 7} .\end{array}$ & $\begin{array}{l}2040 \\
1350 \\
1490\end{array}$ & $\begin{array}{l}1870 \\
1290 \\
1410\end{array}$ & $\begin{array}{l}8,3 \\
4,4 \\
5,4\end{array}$ & $\begin{array}{l}41 \\
45 \\
31\end{array}$ & $\begin{array}{l}21,9 \\
34,9 \\
22,0\end{array}$ & $\begin{array}{l}1,530 \\
1,640 \\
1,364\end{array}$ \\
\hline \multicolumn{3}{|c|}{ Durchschnitt } & 6,0 & & 26,3 & 1,511 \\
\hline
\end{tabular}

Falls 1 bzw. $3 \mathrm{ccm}$ Alkohol pro kg mit der Glukose gleichzeitig zugesetzt wurde, betrug das Leberglykogen je $0,720 \mathrm{bzw}$. 0,123\% (Tabelle 20 und 21). Also erleidet das Glykogen der Leber durch Alkoholzusatz so hochgradig, dass es bei der Anwendung von $3 \mathrm{ccm}$ pro $\mathrm{kg}$ über 10 mal mehr reduziert wurde als bei Tieren ohne Alkoholzusatz.

Tabelle 20.

Derselbe Versuch. 4 Stunden nach $5 \mathrm{~g}$ Glukose und $1 \mathrm{ecm}$ Alkohol pro $\mathrm{kg}$.

\begin{tabular}{|c|c|c|c|c|c|c|}
\hline \multirow[b]{2}{*}{$\begin{array}{c}\text { Versuchs- } \\
\text { tier- } \\
\text { Nr. }\end{array}$} & \multicolumn{2}{|c|}{ Körpergewicht(g) } & \multirow{2}{*}{$\begin{array}{l}\text { Korper- } \\
\text { gewichts- } \\
\text { abnahme } \\
(\%)\end{array}$} & \multicolumn{3}{|c|}{ Leber } \\
\hline & $\begin{array}{l}\text { vor Chloro- } \\
\text { form }\end{array}$ & $\begin{array}{c}\text { vor } \\
\text { Glukose- } \\
\text { Alkohol }\end{array}$ & & $\begin{array}{l}\text { Gewicht } \\
\text { (g) }\end{array}$ & $\begin{array}{c}\text { pro kg K. G. } \\
(\mathrm{g})\end{array}$ & $\begin{array}{c}\text { Glykogen } \\
(\%)\end{array}$ \\
\hline 58 & 2170 & 2030 & 6,5 & 57 & 28,0 & 0,870 \\
\hline 59 & 1670 & 1520 & 9,0 & 53 & 31,7 & 0,610 \\
\hline 60 & 1580 & 1410 & 10,8 & 50 & 31,7 & 0,680 \\
\hline \multicolumn{3}{|c|}{ Durchschnitt } & 8,8 & & 30,5 & 0,720 \\
\hline
\end{tabular}

Tabelle 21.

Derselbe Versuch. 4 Stunden nach $5 \mathrm{~g}$ Glukose und $3 \mathrm{ccm}$ Alkohol pro $\mathrm{kg}$.

\begin{tabular}{|c|c|c|c|c|c|c|}
\hline \multirow{2}{*}{$\begin{array}{c}\text { Versuchs- } \\
\text { tier- } \\
\text { Nr. }\end{array}$} & \multicolumn{2}{|c|}{ Körpergewicht(g) } & \multirow{2}{*}{$\begin{array}{l}\text { Körper- } \\
\text { gewichts- } \\
\text { abnahme } \\
(\%)\end{array}$} & \multicolumn{3}{|c|}{ Leber } \\
\hline & $\begin{array}{l}\text { vor Chloro- } \\
\text { form }\end{array}$ & $\begin{array}{l}\text { vor } \\
\text { Glukose- } \\
\text { Alkohol }\end{array}$ & & $\begin{array}{l}\text { Gewicht } \\
\text { (g) }\end{array}$ & $\begin{array}{c}\text { pro kg K. G. } \\
\text { (g) }\end{array}$ & $\begin{array}{l}\text { Glykogen } \\
(\%)\end{array}$ \\
\hline 61 & 1940 & 1790 & 7,7 & 46 & 25,7 & 0,010 \\
\hline 62 & 1840 & 1740 & 5,4 & 49 & 28,2 & 0,360 \\
\hline 63 & 1600 & 1400 & 12,5 & 42 & 30,0 & 0 \\
\hline \multicolumn{3}{|c|}{ Durchschnitt } & 8,5 & & 28,0 & 0,123 \\
\hline
\end{tabular}

3. Versuche mit $0,25 \mathrm{~cm}$ Chloroform pro $\mathrm{kg}$.

Die Versuchsanordnung gestaltete sich ganz vorschriftsmässig wie beim vorausgeschickten Versuch, abgesehen von $0,25 \mathrm{ccm}$ Chloro- 
form pro $\mathrm{kg}$ statt $0,15 \mathrm{ccm}$. Bei Glukose allein fand ich durchschnittlich 1,082\% Leberglykogen, dagegen bei Zusatz von $1 \mathrm{ccm}$ bzw. $3 \mathrm{ccm}$ Alkohol pro kg zu Glukose nur 0,433 bzw. 0,162\% (Tabelle 22,23 und 24). Es scheint, dass der letzte Wert etwas grösser ist als der von den Tieren, die mit $0,15 \mathrm{ccm}$ Chloroform vergiftet waren. Da man aber bei genauer Durchmusterung der einzelnen Daten bei 2 von 3 Tieren keinen Glykogenvorrat in der Leber finden kann, so entsteht praktisch keine Differenz zwischen beiden Gruppen.

\section{Tabelle 22.}

Glykogengehalt der Leber der mit 0,25 ccm Chloroform pro $\mathrm{kg}$ vergifteten Tiere. 4 Stunden nach $5 \mathrm{~g}$ Glukose pro $\mathrm{kg}$.

\begin{tabular}{|c|c|c|c|c|c|c|}
\hline \multirow{2}{*}{$\begin{array}{c}\text { Versuchs- } \\
\text { tier- } \\
\text { Nr. }\end{array}$} & \multicolumn{2}{|c|}{ Körpergewicht (g) } & \multirow{2}{*}{$\begin{array}{l}\text { Körper- } \\
\text { gewichts- } \\
\text { abnahme } \\
(\%)\end{array}$} & \multicolumn{3}{|c|}{ Leber } \\
\hline & $\begin{array}{c}\text { vor Chloro- } \\
\text { form }\end{array}$ & $\begin{array}{c}\text { vor } \\
\text { Glukose }\end{array}$ & & $\begin{array}{c}\text { Gewicht } \\
\text { (g) }\end{array}$ & $\begin{array}{c}\text { pro kg K. G. } \\
(\mathrm{g})\end{array}$ & $\begin{array}{c}\text { Glykogen } \\
(\%)\end{array}$ \\
\hline $\begin{array}{l}64 \\
65 \\
66\end{array}$ & $\begin{array}{l}2260 \\
1830 \\
2400\end{array}$ & $\begin{array}{l}1980 \\
1690 \\
2240\end{array}$ & $\begin{array}{r}12,4 \\
7,7 \\
6,7\end{array}$ & $\begin{array}{l}75 \\
56 \\
78\end{array}$ & $\begin{array}{l}37,8 \\
33,2 \\
34,8\end{array}$ & $\begin{array}{l}0,964 \\
1,378 \\
0,904\end{array}$ \\
\hline \multicolumn{3}{|c|}{ Durchschnitt } & 8,9 & & 35,3 & 1,082 \\
\hline
\end{tabular}

Tabelle 23.

Derselbe Versuch. 4 Stunden nach $5 \mathrm{~g}$ Glukose und $1 \mathrm{ccm}$ Alkohol pro $\mathrm{kg}$.

\begin{tabular}{|c|c|c|c|c|c|c|}
\hline \multirow[b]{2}{*}{$\begin{array}{c}\text { Versuchs- } \\
\text { tier- } \\
\text { Nr. }\end{array}$} & \multicolumn{2}{|c|}{ Körpergewicht $(g)$} & \multirow{2}{*}{$\begin{array}{c}\text { Körper- } \\
\text { gewichts- } \\
\text { abnahme } \\
(\%)\end{array}$} & \multicolumn{3}{|c|}{ Leber } \\
\hline & $\begin{array}{l}\text { vor Chloro- } \\
\text { form }\end{array}$ & $\begin{array}{c}\text { yor } \\
\text { Glukose- } \\
\text { Alkohol }\end{array}$ & & $\begin{array}{l}\text { Gewicht } \\
\text { (g) }\end{array}$ & $\begin{array}{c}\text { pno kg K. G. } \\
(\mathrm{g})\end{array}$ & $\begin{array}{c}\text { Glykogen } \\
(\%)\end{array}$ \\
\hline $\begin{array}{l}67 \\
68 \\
69\end{array}$ & $\begin{array}{l}2230 \\
2070 \\
2140\end{array}$ & $\begin{array}{l}2100 \\
1950 \\
2030\end{array}$ & $\begin{array}{l}5,8 \\
5,8 \\
5,1\end{array}$ & $\begin{array}{l}\mathbf{5 7} \\
52 \\
76 \\
\end{array}$ & $\begin{array}{l}27,1 \\
26,6 \\
37,4\end{array}$ & $\begin{array}{l}0,300 \\
0,377 \\
0,622 \\
\end{array}$ \\
\hline \multicolumn{3}{|c|}{ Durehschnitt } & 5,6 & & 30,4 & $0, \mathbf{4 3 3}$ \\
\hline
\end{tabular}

Tabelle 24.

Derselbe Versuch. 4 Stunden nach $5 \mathrm{~g}$ Glukose und $3 \mathrm{ccm}$ Alkohol pro $\mathrm{kg}$.

\begin{tabular}{|c|c|c|c|c|c|c|}
\hline \multirow[b]{2}{*}{$\begin{array}{c}\text { Versuchs- } \\
\text { tier- } \\
\text { Nr. }\end{array}$} & \multicolumn{2}{|c|}{ Körpergewicht(g) } & \multirow{2}{*}{$\begin{array}{l}\text { Körper- } \\
\text { gewichts- } \\
\text { abnahme } \\
(\%)\end{array}$} & \multicolumn{3}{|c|}{ Leber } \\
\hline & $\begin{array}{l}\text { vor Chloro- } \\
\text { form }\end{array}$ & $\begin{array}{c}\text { vor } \\
\text { Glukose- } \\
\text { Alkohol }\end{array}$ & & $\begin{array}{l}\text { Gewicht } \\
\text { (g) }\end{array}$ & pro $\underset{(\mathrm{gg})}{\mathrm{kg} . \mathrm{G} .}$ & $\begin{array}{c}\text { Glykogen } \\
(\%)\end{array}$ \\
\hline $\begin{array}{r}70 \\
71 \\
72 \\
\end{array}$ & $\begin{array}{l}1800 \\
1470 \\
2180 \\
\end{array}$ & $\begin{array}{l}1630 \\
1350 \\
2030 \\
\end{array}$ & $\begin{array}{l}9,5 \\
8,2 \\
6,9 \\
\end{array}$ & $\begin{array}{l}45 \\
49 \\
65 \\
\end{array}$ & $\begin{array}{l}28,6 \\
36,3 \\
32,0 \\
\end{array}$ & $\begin{array}{l}0 \\
0,486 \\
0 \\
\end{array}$ \\
\hline \multicolumn{3}{|c|}{ Durchschnitt } & 8,2 & & 32,3 & 0,162 \\
\hline
\end{tabular}


Aus den oben erwähnten Tatsachen kann man wohl schliessen, dass der Alkoholzusatz bei Glukosebehandlung unter derartigen Leberschädigungen ohne Zweifel schädlich wirkt.

\section{Besprechung der Ergebnisse.}

Wie ich eingangs ausführlich erörtert habe, wird der Alkohol heute auf ausgedehntem Gebiet angewandt, ohne dass man weiss, ob es bei einem gewissen Krankheitszustande nicht nur vergeblich, sondern mehr schädigend wirkt. Die vorliegende Untersuchung ist darauf gerichtet, ob der Alkohol bei verschiedenen experimentellen Leberschädigungen tatsächlich ohne Schaden angewandt werden kann, besonders bei kombinierter Anwendung von Glukose.

Um unbestimmte Leberschäden zu schaffen, zog ich zum Versuch 3 Arten von Giften, d.i. Typhusvakzin, Diphtherietoxin und Chloroform heran. Ausser allgemeiner Vergiftung rufen alle diese Gifte eine gewisse pathologische Veränderung in parenchymatösen Organen, besonders in der Leber hervor. Mit einer gewissen Dose der Gifte kann man einen beträchtlichen Glykogenschwund aus der Leber auslösen und dabei durch Glukosegabe eine merkliche Glykogenablagerung wieder dort erzielen.

Hier handelt es sich nun um die Beziehung des Alkohols zum Glykogendepot bzw. zur Glukoseassimilation. Die durchschnittlichen Daten darüber aus meinen drei Versuchsreihen gebe ich hier in Tabelle 25 zusammenfassend wieder. Wie in dieser Tafel gezeigt, beeinträchtigt der Alkohol mehr oder weniger die Ablagerung des Glykogens in der Leber und verursacht eine Verarmung desselben.

\section{Tabelle 25.}

Zusammenfassende Darstellung von den Veränderungen des Leberglykogens:

\begin{tabular}{|c|c|c|c|c|}
\hline \multirow[b]{2}{*}{ Art des Giftes } & \multirow[b]{2}{*}{$\begin{array}{c}\text { Zugeführte } \\
\text { Giftdose } \\
\text { (pro kg) }\end{array}$} & \multicolumn{3}{|c|}{ Probemahlzeit (pro kg) } \\
\hline & & $\begin{array}{c}5 \mathrm{~g} \text { Glukose } \\
\text { allein }\end{array}$ & $\begin{array}{c}5 \mathrm{~g} \text { Glukose } \\
\text { und } \\
1 \mathrm{ccm} \text { Alkohol }\end{array}$ & $\begin{array}{c}5 \mathrm{~g} \text { Glukose } \\
\text { und } \\
3 \mathrm{ccm} \text { Alk ohol }\end{array}$ \\
\hline Typhusvakzin & $\begin{array}{c}\text { Kontrolle } \\
0,5 \mathrm{ccm} \\
1,0 \mathrm{ccm}\end{array}$ & $\begin{array}{l}2,950 \\
2,177 \\
1,018\end{array}$ & $\begin{array}{l}1,278 \\
0,997 \\
0,847\end{array}$ & $\begin{array}{l}0,869 \\
0,520 \\
0,492\end{array}$ \\
\hline $\begin{array}{l}\text { 500fach verdünntes } \\
\text { Diphtherietoxin }\end{array}$ & $\begin{array}{c}\text { Kontrolle } \\
0,3 \mathrm{ccm} \\
0,7 \mathrm{~cm}\end{array}$ & $\begin{array}{l}2,950 \\
1,298 \\
1,208\end{array}$ & $\begin{array}{l}1,278 \\
0,923 \\
0,844 \\
\end{array}$ & $\begin{array}{l}0,869 \\
0,819 \\
0,730\end{array}$ \\
\hline Chloroform & $\begin{array}{l}\text { Kontrolle } \\
0,15 \mathrm{ccm} \\
0,25 \mathrm{ccm}\end{array}$ & $\begin{array}{l}2,600 \\
1,511 \\
1,082\end{array}$ & $\begin{array}{l}2,106 \\
0,720 \\
0,433\end{array}$ & $\begin{array}{l}0,935 \\
0,123 \\
0,162\end{array}$ \\
\hline
\end{tabular}


Hier kann man ungezwungen zu der Annahme kommen, dass der Alkohol bei verschiedenen Leberschäden eine Gegenwirkung gegen die Glukosetherapie besitzt, und dass die Verminderung des Glykogenvorrats sicherlich durch Zunahme der Alkoholmenge verstärkt wird. Wenn eine Alkoholtherapie mit grösserer Menge auszuführen ist, muss man sehr vorsichtig den Leberzustand des Organismus berücksichtigen.

Aus dem Ergebnisse vorliegender Untersuchungen scheint es mir ganz richtig zu sein, dass man bei Leberleiden, besonders akuten Infektionskrankheiten, wobei immer mehr oder minder grosse Leberschädigung einhergeht, die Anwendung einer grösseren Dose Alkohols streng meiden soll.

\section{Zusammenfassung.}

1. Die Glykogenneubildung aus Glukose bei mit Typhusvakzin, Diphtherietoxin oder Chloroform vergifteten Kaninchen wird durch Hinzufügung von Alkohol so stark verhindert, dass die zugeführte Glukose gelegentlich, z.B. bei Chloroformtieren, in der Leber kaum assimiliert werden kann.

2. Daher ist der Alkohol bei Leberleiden, wahrscheinlich auch bei akuten Infektionskrankheiten streng abzuhalten.

\section{Literatur.}

(1) M a tun a ga, Tōhoku J. Exper. Med., 1942, 44,

(2) Thursz, Z. Krebsf., 1928, 26, 260.

(3) Landau, Feijin u. Ba uer, Presse méd., 1931, 39, 523.

(4) Marin o, Gi. Clin. Med., 1934, 15, 1554.

(5) B rü 11, Med. Klin., 1934, 538 u. 576.

(6) N a g a matra, Zikken ihō, 1934, Nr. 240, 1751.

(7) Ki n, Tyōsen Thō, 1934, 4, 85 ; Rinsyō Naika., 1935, 3, 37 ; Tyōsen Igaku Zassi, $1935,25,1598$.

(8) Wa ta na be a. S a no, Zittiika to Rinsyō, 1935, 12, 633.

(9) Akaiwa u. Suzuki,-Terapi, 1937, 14, 279.

(10) Gotō, Tiryō Gaku Zassi, 1937, 7, 1347.

(11) A z u ma, Zikken Thō, 1938, 280, 570.

(12) A zuma u. Matan o, Tiryō oyobi Syohō, 1938, 220, 1104.

(13). I was a ki u. S in nō, Ōsaka Izi Sinsi, 1938, 1278.

(14) W a t a na be, Tōhoku Igaku Zassi, 1941, 28, 1055.

(15) Hotta, Tōhoku J. Exper. Med., 1932, 20, 65 u. 211.

(16) Naga o, ebenda, 1934, 24, 529.

(17) Ts ud a, ebenda, 1935, 25, 80.

(18) Sim i zu, ebenda, 1935, 36, 211. 